\title{
INVARIANT, DECOUPLING AND BLOCKING ZEROS OF FRACTIONAL LINEAR SYSTEMS
}

\author{
Tadeusz KACZOREK ${ }^{*}$ \\ *Faculty of Electrical Engineering, Białystok University of Technology, ul. Wiejska 45D, 15-351 Białystok, Poland \\ kaczorek@ee.pw.edu.pl
}

received 14 June 2017, revised 1 March 2018, accepted 6 March 2018

\begin{abstract}
The notions of invariant, decoupling and blocking zeros are extended to the fractional linear systems. It is shown that: 1) The zeros are closely connected with the controllability and observability of the linear systems and their transfer functions matrices. 2) The state vector of the fractional system for any input and zero initial conditions is independent of the input decoupling zeros of the system. 3) The output of the fractional system for any input and zero initial conditions is independent of the input-output decoupling zeros of the system.
\end{abstract}

Key words: Fractional, Linear, System, Invariant, Decoupling, Blocking Zero

\section{INTRODUCTION}

The notion of controllability and observability and the decomposition of linear systems have been introduced by Kalman (1960, 1963). These notions are the basic concepts of the modern control theory (Antsaklis and Michel, 2006; Kaczorek, 1993; Kailath, 1980; Rosenbrock, 1970; Wolovich, 1974; Valcher, 1977). They have been also extended to positive linear systems (Farina and Rinaldi, 2000; Kaczorek, 2011a). The positive circuits and their reachability has been investigated in (Kaczorek, 2011a; Kaczorek and Rogowski, 2015) and controllability and observability of electrical circuits in (Kaczorek, 2011c; Kaczorek and Rogowski, 2015).

The reachability of linear systems is closely related to the controllability of the systems. Specially for positive linear systems, the conditions for the controllability are much stronger than for the reachability (Kaczorek, 2002, 2016). Tests for the reachability and controllability of standard and positive linear systems are given in Kaczorek $(2002,2008)$ and in Kaczorek and Rogowski (2015). The positivity and reachability of fractional continuous-time linear systems and electrical circuits have been addressed in (Kaczorek, 2008, 2011a, 2013d; Kaczorek and Rogowski, 2015). The finite zeros of positive discrete-time and continuous-time linear systems have been investigated in Tokarzewski (2011a, 2011b) and the decoupling zeros of positive discrete-time linear systems and positive electrical circuits in Kaczorek (2010, 2013b).

The positive linear systems consisting of $n$ subsystems with different fractional orders has been analyzed in Kaczorek (2011b). Some recent interesting results in the fractional systems theory and its applications can be found in Dzieliński Sierociuk and Sarwas (2009) and in Kaczorek (2017b). The constructability and observability of standard and positive electrical circuits in Kaczorek (2013a). The stability of fractional systems has been analysed in (Busłowicz, 2008; Dzieliński and Sierociuk, 2008; Kaczorek, 2008, 2009).

The invariant, decoupling and blocking zeros of linear positive systems and electrical circuits have been addressed in Kaczorek (1993, 2017b).

In this paper the notions of invariant zeros, decoupling zeros and blocking zeros of linear systems will be extended to the fractional linear systems. The paper is organized as follows. In section 2 the elementary operations and Smith canonical form of polynomial matrices are recalled. Basic definitions and theorems concerning the invariant, decoupling and blocking zeros of fractional linear systems are presented in section 3. Concluding remarks are given in section 4 .

The following notation will be used: $\mathfrak{R}$ is the set of real numbers, $\Re^{\mathrm{n} \times \mathrm{m}}$ represents the set of $\mathrm{n} \times \mathrm{m}$ real matrices, $\mathfrak{R}^{\mathrm{n} \times \mathrm{m}}[\mathrm{s}]$ denotes the set of $n \times m$ polynomial matrices with real coefficients, $\mathrm{C}$ is the field of complex numbers, $\mathrm{I}_{\mathrm{n}}$ is the $\mathrm{n} \times \mathrm{n}$ identity matrix.

\section{ELEMENTARY OPERATIONS AND SMITH CANONICAL FORM OF POLYNOMIAL MATRICES}

Definition 1. (Kaczorek, 1993) The following operations on polynomial matrices are called elementary row (column) operations:

- Multiplication of the i-th row (column) by scalar (number) $\mathrm{c}$. This operation will be denoted by $\mathrm{L}(\mathrm{i} \times \mathrm{c})(\mathrm{R}(\mathrm{i} \times \mathrm{c}))$.

- Addition to the i-th row (column) of the j-th row (column) multiplied by any polynomial $b(s)$. This operation will be denoted by $\mathrm{L}(\mathrm{i}+\mathrm{j} \times \mathrm{b}(\mathrm{s}))(\mathrm{R}(\mathrm{i}+\mathrm{j} \times \mathrm{b}(\mathrm{s})))$.

- Intercharge of the $i$-th and $j$-th rows (columns). This operations will be denoted by $L(i, j)(R(i, j))$.

Applying the elementary row and column operations to identity matrices we obtain unimodulary matrices. The elementary row (column) operations are equivalent to premultiplication (postmultiplication) of the matrix by suitable unimodular matrices (Kaczorek 1993). The elementary row and column operations do not change the rank of the matrices. 
Definition 2. (Gantmacher, 1988, Kaczorek, 1993) The polynomial matrix:

$A_{S}(s)=\left[\begin{array}{lllllll}a_{1}(s) & 0 & \cdots & 0 & 0 & \cdots & 0 \\ 0 & a_{2}(s) & \cdots & 0 & 0 & \cdots & 0 \\ \vdots & \vdots & \ddots & \vdots & \vdots & \ddots & \vdots \\ 0 & 0 & \cdots & a_{r}(s) & 0 & \cdots & 0 \\ 0 & 0 & \cdots & 0 & 0 & \cdots & 0 \\ \vdots & \vdots & \ddots & \vdots & \vdots & \ddots & \vdots \\ 0 & 0 & \cdots & 0 & 0 & \cdots & 0\end{array}\right]$

$\in \mathfrak{R}^{m \times p}[s]$

is called the Smith canonical form of the polynomial matrix $\mathrm{A}(\mathrm{s}) \in \mathfrak{R}^{\mathrm{m} \times \mathrm{p}}[\mathrm{s}]$, where $\mathrm{a}_{1}(\mathrm{~s}), \mathrm{a}_{2}(\mathrm{~s}), \ldots, \mathrm{a}_{\mathrm{r}}(\mathrm{s})$ are nonzero invariant polynomials (with leading coefficients equal to 1) of the matrix $A(s)$ such that $a_{i}(s) \mid a_{i+1}(s)\left(a_{i}(s)\right.$ divides $a_{i+1}(s)$ with zero remainder) for $\mathrm{i}=1, \ldots, \mathrm{r}-1$ and $\mathrm{r}=\operatorname{rankA}(\mathrm{s})$.

The invariant polynomials $a_{1}(s), a_{2}(s), \ldots, a_{r}(s)$ of the matrix $A(s)$ are uniquely determined by:

$a_{k}(s)=\frac{D_{k}(s)}{D_{k-1}(s)}$ for $k=1, \ldots, r\left(D_{0}(s)=1\right)$,

where $\mathrm{D}_{\mathrm{k}}(\mathrm{s})$ is the greatest common divisor of all of the $\mathrm{k} \times \mathrm{k}$ minors of the matrix $A(s)$.

The equivalent polynomial matrices have the same greatest common divisors $\mathrm{D}_{\mathrm{k}}(\mathrm{s})$ (Kaczorek, 1993).

\section{INVARIANT, DECOUPLING AND BLOCKING ZEROS OF FRACTIONAL LINEAR SYSTEMS}

Consider the fractional linear system (Kaczorek, 2011d):

$\frac{d^{\alpha} x(t)}{d t^{\alpha}}=A x(t)+B u(t), 0<\alpha<1$

$y=C x$,

where:

${ }_{0} D_{t}^{\alpha} f(t)=\frac{d^{\alpha} f(t)}{d t^{\alpha}}=\frac{1}{\Gamma(1-\alpha)} \int_{0}^{t} \frac{\dot{f}(\tau)}{(t-\tau)^{\alpha}} d \tau$,

is Caputo definition of the fractional derivative of a order, $\dot{\mathrm{f}}(\tau)=\frac{\mathrm{df}(\tau)}{\mathrm{d} \tau}, \Gamma(\mathrm{x})=\int_{0}^{\infty} \mathrm{t}^{\mathrm{x}-1} \mathrm{e}^{-\mathrm{t}} \mathrm{dt}, \operatorname{Re}(\mathrm{x})>0$ is the Euler gamma function and $\mathrm{x}=\mathrm{x}(\mathrm{t}) \in \mathfrak{R}^{\mathrm{n}}, \mathrm{u}=\mathrm{u}(\mathrm{t}) \in \mathfrak{R}^{\mathrm{m}}$, $\mathrm{y}=\mathrm{y}(\mathrm{t}) \in \mathfrak{R}^{\mathrm{p}}$ are the state, input and output vectors and $\mathrm{A} \in \mathfrak{R}^{\mathrm{n} \times \mathrm{n}}, \mathrm{B} \in \mathfrak{R}^{\mathrm{n} \times \mathrm{m}}, \mathrm{C} \in \mathfrak{R}^{\mathrm{p} \times \mathrm{n}}$.

The system matrix of the linear system (3) is defined by:

$S(\lambda)=\left[\begin{array}{ll}I_{n} \lambda-A & B \\ C & 0\end{array}\right] \in \Re^{(n+p) \times(n+m)}[\lambda], \lambda=s^{\alpha}$

Let the matrix:

$S_{S}(\lambda)=\left[\begin{array}{llll}\operatorname{diag}\left[p_{1}(\lambda)\right. & \cdots & \left.p_{r}(\lambda)\right] & 0 \\ 0 & & & 0\end{array}\right] \in$ $\mathfrak{R}^{(n+p) \times(n+m)}[\lambda]$

be the canonical Smith form of the system matrix (4), where $\mathrm{p}_{1}(\lambda), \ldots, \mathrm{p}_{\mathrm{r}}(\lambda)$ are the invariant polynomials satisfying the condition $\mathrm{p}_{\mathrm{i}}(\lambda) \mid \mathrm{p}_{\mathrm{i}+1}(\lambda) \quad$ for $\mathrm{i}=1, \ldots, \mathrm{r}-1$ and $r=\operatorname{rankS}(\lambda)$.

The invariant polynomials are determined by:
$p_{k}(\lambda)=\frac{D_{k}(\lambda)}{D_{k-1}(\lambda)}$ for $k=1, \ldots, r\left(D_{0}(\lambda)=1\right)$.

From (6) we have:

$p(\lambda)=p_{1}(\lambda) \ldots p_{r}(\lambda)=\frac{D_{1}(\lambda)}{D_{0}(\lambda)} \frac{D_{2}(\lambda)}{D_{1}(\lambda)} \ldots \frac{D_{r}(\lambda)}{D_{r-1}(\lambda)}=D_{r}(\lambda)$.

The polynomial $p(\lambda)$ is called the invariant zero polynomial of the system (3).

Definition 3. The zero of the polynomial $p(\lambda)$ is called the invariant zero of the system (3.1).

Theorem 1. (Kaczorek, 1993) If $m=p$ and the matrix (4) has full rank then:

$p(\lambda)=\operatorname{det} S_{S}(\lambda)=c \operatorname{det} S_{S}(\lambda)$,

where $c=\operatorname{det} L(\lambda) \operatorname{det} R(\lambda)$ since $L(\lambda)$ and $R(\lambda)$ are unimodular matrices of elementary row and column operations.

Theorem 2. If $\mathrm{m}=\mathrm{p}$ then:

$p(\lambda)=\operatorname{det}\left[\begin{array}{ll}I_{n} \lambda-A & -B \\ C & 0\end{array}\right]=\operatorname{det}\left[I_{n} \lambda-A\right] \operatorname{det} T(\lambda)$,

where:

$T(\lambda)=C\left[I_{n} \lambda-A\right]^{-1} B$.

Proof. It is easy to see that:

$\left[\begin{array}{ll}I_{n} & 0 \\ -C\left[I_{n} \lambda-A\right]^{-1} & I_{p}\end{array}\right]\left[\begin{array}{ll}I_{n} \lambda-A & -B \\ C & 0\end{array}\right]$

$=\left[\begin{array}{ll}I_{n} \lambda-A & -B \\ 0 & T(\lambda)\end{array}\right]$

and:

$\operatorname{det}\left\{\left[\begin{array}{ll}I_{n} & 0 \\ -C\left[I_{n} \lambda-A\right]^{-1} & I_{p}\end{array}\right]\left[\begin{array}{ll}I_{n} \lambda-A & -B \\ C & 0\end{array}\right]\right\}$

$=\operatorname{det}\left[\begin{array}{ll}I_{n} \lambda-A & -B \\ 0 & T(\lambda)\end{array}\right]$

since:

$\operatorname{det}\left[\begin{array}{ll}I_{n} & 0 \\ -C\left[I_{n} \lambda-A\right]^{-1} & I_{p}\end{array}\right]=1$.

Consider the submatrix:

$S_{1}(\lambda)=\left[\begin{array}{ll}I_{n} \lambda-A & B\end{array}\right]$

of the system matrix (4).

Definition 4. (Kaczorek, 1993) A number $\mathrm{z} \in \mathrm{C}$ for which:

$\operatorname{rank}\left[I_{n} Z-A \quad B\right]<n$

is called the input decoupling (i.d.) zero of the fractional system (3).

Let the matrix:

$S_{1 S}(\lambda)=\left[\operatorname{diag}\left[\bar{p}_{1}(\lambda) \quad \cdots \quad \bar{p}_{n}(\lambda)\right] \quad 0\right] \in \mathfrak{R}^{n \times(n+m)}[\lambda]$

be the canonical Smith form of the matrix (13).

Note that $z \in C$ is an i.d. zero of the system (3) if and only if $z$ is a zero of the polynomial:

$\bar{p}(\lambda)=\bar{p}_{1}(\lambda) \ldots \bar{p}_{n}(\lambda)$. 
Therefore, the i.d. zeros of the system are the zeros of the polynomial (16). The system has no i.d. zeros if and only if $\bar{p}(\lambda)=1$, i.e. the matrix $S_{1}(\lambda)$ has the canonical Smith form $\left[\begin{array}{ll}I_{n} & 0\end{array}\right]$. The i.d. zeros represent unreachable modes of the system (3) (Kaczorek, 1993).

The number of i.d. zeros $\mathrm{n}_{1}$ of the system (3) is equal to the rank defect of its reachability (controllability) matrix, i.e.

$n_{1}=n-\operatorname{rank} R_{n}$,

where:

$R_{n}=\left[\begin{array}{llll}B & A B & \cdots & A^{n-1} B\end{array}\right]$.

Theorem 3. (Kaczorek 1993) The state vector $x$ of the fractional system (3) for any input $u(t)$ and zero initial state $x(0)=0$ is independent of the i.d. zeros of the system.

Consider the submatrix:

$S_{2}(\lambda)=\left[\begin{array}{l}I_{n} \lambda-A \\ C\end{array}\right]$

of the system matrix (4).

Definition 5. (Kaczorek 1993) A number $\mathrm{z} \in \mathrm{C}$ for which:

$\operatorname{rank}\left[\begin{array}{l}I_{n} Z-A \\ C\end{array}\right]<n$

is called the output-decoupling (o.d.) zero of the fractional system (3).

Let the matrix:

$S_{2 S}(\lambda)=\left[\begin{array}{lll}\operatorname{diag}\left[\hat{p}_{1}(\lambda)\right. & \cdots & \left.\hat{p}_{n}(\lambda)\right] \\ 0 & & \end{array}\right] \in \Re^{(n+p) \times n}[\lambda]$

be the canonical Smith form of the matrix (19).

Note that $z \in C$ is an o.d. zero of the system (3) if and only if $z$ is a zero of the polynomial:

$\hat{p}(\lambda)=\hat{p}_{1}(\lambda) \ldots \hat{p}_{n}(\lambda)$.

Therefore, the o.d. zeros of the system are the zeros of the polynomial (22). The system has no o.d. zeros if and only if $\hat{p}(\lambda)=1$, i.e. the matrix $S_{2}(\lambda)$ has the canonical Smith form $\left[\begin{array}{c}\mathrm{I}_{\mathrm{n}} \\ 0\end{array}\right]$. The o.d. zeros represent unobservable modes of the system (3) (Kaczorek, 1993).

The number of o.d. zeros $n_{2}$ of the system (3) is equal to the rank defect of its observability matrix, i.e.

$n_{2}=n-\operatorname{rank} O_{n}$,

where:

$$
O_{n}=\left[\begin{array}{l}
C \\
C A \\
\vdots \\
C A^{n-1}
\end{array}\right] .
$$

Theorem 4. (Kaczorek, 1993) The output $y$ of the fractional system (3) for any input $\mathrm{u}^{\prime}(\mathrm{t})=\mathrm{Bu}(\mathrm{t})$ and zero initial condition $\mathrm{x}(0)=0$ is independent of the o.d. zeros of the system.

Definition 6. (Kaczorek, 1993) A number $\mathrm{z} \in \mathrm{C}$ for which both conditions (14) and (20) are satisfied is called the input-output decoupling (i.o.d.) zero of the fractional system (3).

Therefore, $\mathrm{z} \in \mathrm{C}$ is an i.o.d. zero if and only if it is both an i.d. zero and an o.d. zero of the system.

The number of i.o.d. zeros $n_{i o}$ of the fractional system (3) is equal to:

$n_{i o}=n-\operatorname{rank} R_{n}-\operatorname{rank} O_{n}+\operatorname{rank} O_{n} R_{n}$.

Definition 7. (Kaczorek, 1993) A number $\mathrm{z} \in \mathrm{C}$ is called a blocking zero of the system (3) if:

$C\left[I_{n} z-A\right]_{a d} B=0$,

where $\left[\mathrm{I}_{\mathrm{n}} \mathrm{Z}-\mathrm{A}\right]_{\mathrm{ad}}$ is the adjoint matrix.

If (26) is satisfied for all $\lambda$ then by definition the system has no blocking zeros.

Theorem 5. (Kaczorek, 1993) A number $\mathrm{z} \in \mathrm{C}$ is an uncontrollable and/or unobservable mode of the system if and only if $z$ is a blocking zero of the system.

Theorem 6 . If the transfer function matrix:

$T(\lambda)=C\left[I_{n} \lambda-A\right]^{-1} B=0$

then:

$O_{n} R_{n}=0$,

where $\mathrm{O}_{\mathrm{n}}$ and $\mathrm{R}_{\mathrm{n}}$ are defined by (24) and (18), respectively.

Proof. Note that if (27) holds then:

$C A^{k} B=0$ for $k=0,1, \ldots, n-1$.

Using (24), (18) and (29) we obtain:

$O_{n} R_{n}=\left[\begin{array}{l}C \\ C A \\ \vdots \\ C A^{n-1}\end{array}\right]\left[\begin{array}{llll}B & A B & \cdots & A^{n-1} B\end{array}\right]$
$=\left[\begin{array}{llll}C B & C A B & \cdots & C A^{n-1} B \\ C A B & C A^{2} B & \cdots & C A^{n} B \\ \vdots & \vdots & \ddots & \vdots \\ C A^{n-1} B & C A^{n} B & \cdots & C A^{2(n-1)} B\end{array}\right]=0$.

This completes the proof. $\square$

Theorem 7. Let for the fractional system (3) the condition (27) be satisfied. Then:

1. the pair $(A, B)$ is unreachable if $C \neq 0$,

2. the pair $(A, C)$ is unobservable if $B \neq 0$.

Proof. From (13) we have:

$C\left[\begin{array}{llll}B & A B & \cdots & A^{n-1} B\end{array}\right]=0$

and:

$\operatorname{rank}\left[\begin{array}{llll}B & A B & \cdots & A^{n-1} B\end{array}\right]<n$

if $C \neq 0$. Therefore, the pair $(A, B)$ is unreachable.

Similarly, from (30) we have:

$\left[\begin{array}{l}C \\ C A \\ \vdots \\ C A^{n-1}\end{array}\right] B=0$

and:

$\operatorname{rank}\left[\begin{array}{l}C \\ C A \\ \vdots \\ C A^{n-1}\end{array}\right]<n$

if $B \neq 0$. Therefore, the pair $(A, C)$ is unobservable. $\square$ 
Example 1. Consider the electrical circuit shown in Fig. 1 with given resistances $\mathrm{R}_{1}, \mathrm{R}_{2}, \mathrm{R}_{3}, \mathrm{R}_{4}$, inductance $L$, capacitance $C$ and voltage source $e$.

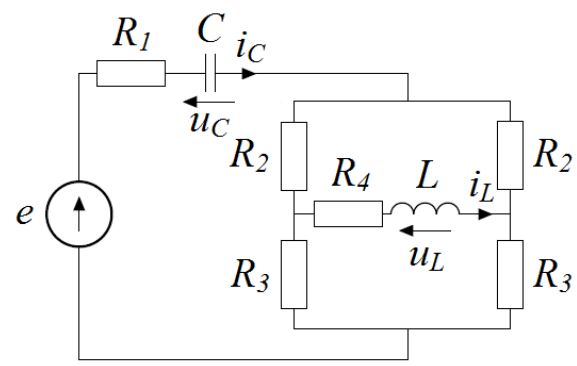

Fig. 1. Electrical circuit of Example 1

Knowing that $i_{c}=C \frac{d^{\alpha} u_{C}}{d t^{\alpha}}, \quad u_{L}=L \frac{d^{\alpha} i_{L}}{d t^{\alpha}}$ and using Kirchhoff's laws we may write the equations:

$e=R C \frac{d^{\alpha} u_{C}}{d t^{\alpha}}+u_{C}, R=R_{1}+\frac{R_{2}+R_{3}}{2}$,

$R_{4} i_{L}+L \frac{d^{\alpha} i_{L}}{d t^{\alpha}}=0$.

As the output $y$ we choose:

$y=i_{L}$.

The equations (35) and (36) can be rewritten in the form:

$\frac{d^{\alpha}}{d t^{\alpha}}\left[\begin{array}{l}u_{C} \\ i_{L}\end{array}\right]=A_{2}\left[\begin{array}{l}u_{C} \\ i_{L}\end{array}\right]+B_{2} e, y=C_{2}\left[\begin{array}{l}u_{C} \\ i_{L}\end{array}\right]$,

where:

$A_{2}=\left[\begin{array}{ll}-\frac{1}{R C} & 0 \\ 0 & -\frac{R_{4}}{L}\end{array}\right], B_{2}=\left[\begin{array}{c}\frac{1}{R C} \\ 0\end{array}\right], C_{2}=\left[\begin{array}{ll}0 & 1\end{array}\right]$.

The transfer function of the electrical circuit is:

$$
\begin{aligned}
& T(\lambda)=C_{2}\left[I_{2} \lambda-A_{2}\right]^{-1} B_{2} \\
& =\left[\begin{array}{ll}
0 & 1
\end{array}\right]\left[\begin{array}{ll}
\lambda+\frac{1}{R C} & 0 \\
0 & \lambda+\frac{R_{4}}{L}
\end{array}\right]^{-1}\left[\begin{array}{c}
\frac{1}{R C} \\
0
\end{array}\right]=0
\end{aligned}
$$

for all values of $R_{1}, R_{2}, R_{3}, R_{4}, L$ and $C$.

The electrical circuit with (37b) is unreachable and unobservable since the matrices:

$$
\begin{aligned}
& R_{2}=\left[\begin{array}{ll}
B_{2} & A_{2} B_{2}
\end{array}\right]=\left[\begin{array}{ll}
\frac{1}{R C} & -\frac{1}{(R C)^{2}} \\
0 & 0
\end{array}\right], \\
& O_{2}=\left[\begin{array}{l}
C_{2} \\
C_{2} A_{2}
\end{array}\right]=\left[\begin{array}{ll}
0 & 1 \\
0 & -\frac{R_{4}}{L}
\end{array}\right]
\end{aligned}
$$

have only one nonzero column and one nonzero row, respectively. From (39) we have:

$O_{2} R_{2}=\left[\begin{array}{ll}0 & 1 \\ 0 & -\frac{R_{4}}{L}\end{array}\right]\left[\begin{array}{ll}\frac{1}{R C} & -\frac{1}{(R C)^{2}} \\ 0 & 0\end{array}\right]=\left[\begin{array}{ll}0 & 0 \\ 0 & 0\end{array}\right]$.
From (14) and (37b) we obtain:

$\operatorname{rank}\left[I_{n} Z-A_{2} \quad B_{2}\right]=\operatorname{rank}\left[\begin{array}{lll}z+\frac{1}{R C} & 0 & \frac{1}{R C} \\ 0 & Z+\frac{R_{4}}{L} & 0\end{array}\right]=1$

Therefore, by Definition 4 the electrical circuit has one inputdecoupling zero $z_{1}=-\frac{R_{4}}{L}$.

From (14) and (37b) we have:

$\operatorname{rank}\left[\begin{array}{l}I_{n} z-A_{2} \\ C_{2}\end{array}\right]=\operatorname{rank}\left[\begin{array}{ll}z+\frac{1}{R C} & 0 \\ 0 & z+\frac{R_{4}}{L} \\ 0 & 1\end{array}\right]=1$

and by Definition 5 the electrical circuit has also one outputdecoupling zero $z_{2}=-\frac{1}{R C}$.

Therefore by Definition 5 the electrical circuit has no inputoutput decoupling zeros since $\mathrm{z}_{1} \neq \mathrm{z}_{2}$.

From (42) it follows that the electrical circuit has no blocking zeros since (38) holds for all $\lambda$.

\section{CONCLUDING REMARKS}

The notions of invariant, decoupling and blocking zeros have been extended to the fractional linear systems. The relationship between the decoupling zeros and the controllability and observability of the fractional linear systems has been shown. It has been also shown that if the transfer matrix is zero then the product of the observability and controllability matrices is zero matrix (Theorem 6) and if the product is zero then the pair $(A, B)$ is unreachable if $\mathrm{C}=0$ and the pair $(\mathrm{A}, \mathrm{C})$ is unobservable if $\mathrm{B}=0$ (Theorem 7). The considerations can be extended to the positive fractional linear systems and electrical circuits.

\section{REFERENCES}

1. Antsaklis E., Michel A. (2006), Linear Systems, Birkhauser, Boston.

2. Busłowicz M. (2008), Stability of linear continuous-time fractional order systems with delays of the retarded type, Bull. Pol. Acad. Sci. Tech., 56(4). 319-324.

3. Dzieliński A., Sierociuk D. (2008), Stability of discrete fractional order state-space systems, Journal of Vibrations and Control, 14(9-10), 1543-1556.

4. Dzieliński A., Sierociuk D., Sarwas G. (2009), Ultracapacitor parameters identification based on fractional order model, Proc. European Control Conference, Budapest, Hungary, 196-200.

5. Farina L., Rinaldi S. (2000), Positive Linear Systems: Theory and Applications, J. Wiley \& Sons, New York.

6. Gantmacher, F.R. (1988), The theory of matrices, AMS Chelsea Publishing, Rhode Island.

7. Kaczorek T. (1993), Linear Control Systems, vol. 1, J. Wiley, New York.

8. Kaczorek T. (2002), Positive 1D and 2D Systems, Springer-Verlag, London.

9. Kaczorek T. (2008), Practical stability of positive fractional discretetime linear systems, Bull. Pol. Acad. Sci. Tech., 56(4), 313-317.

10. Kaczorek T. (2009), Asymptotic stability of positive fractional 2D linear systems, Bull. Pol. Acad. Sci. Tech., 57(3), 289-292.

11. Kaczorek T. (2010), Decoupling zeros of positive discrete-time linear systems, Circuits and Systems, 1, 41-48. 
12. Kaczorek T. (2011a), Positive electrical circuits and their reachability, Archives of Electrical Engineering, 60(3), 283-301.

13. Kaczorek T. (2011b), Positive linear systems consisting of $n$ subsystems with different fractional orders, IEEE Trans. Circuits and Systems, 58(6), 1203-1210.

14. Kaczorek T. (2011c), Positivity and reachability of fractional electrical circuits, Acta Mechanica et Automatica, 5(2), 42-51.

15. Kaczorek T. (2011d), Selected Problems of Fractional Systems Theory, Springer-Verlag, Berlin.

16. Kaczorek T. (2013a), Constructability and observability of standard and positive electrical circuits, Electrical Review, 89(7), 132-136.

17. Kaczorek T. (2013b), Decoupling zeros of positive electrical circuits, Archives of Electrical Engineering, 62(4), 553-568.

18. Kaczorek T. (2017a), Invariant, decoupling and blocking zeros of positive linear electrical circuits with zero transfer matrices, Circuits, Systems, and Signal Processing, 36(11), 4716-4728.

19. Kaczorek T. (2017b), Specific properties of invariant, decoupling and blocking zeros of positive linear electrical circuits with zero transfer matrices, Proc. of SPIE, $104451 \mathrm{C}$.

20. Kaczorek T., Rogowski K. (2015), Fractional Linear Systems and Electrical Circuits, Studies in Systems, Decision and Control, vol. 13, Springer.

21. Kailath T. (1980), Linear systems, Prentice Hall, Englewood Cliffs, New York.
22. Kalman R. (1960), On the general theory of control systems, Proc. First Intern. Congress on Automatic Control, London, UK: Butterworth, 481-493.

23. Kalman R. (1963), Mathematical description of linear systems, SIAM J. Control, 1(2), 152-192.

24. Rosenbrock H. (1970), State-space and multivariable theory, J. Wiley, New York.

25. Tokarzewski J. (2011a), Finite zeros of positive linear discrete-time systems, Bull. Pol. Acad. Sci. Tech., 59(3), 287-292.

26. Tokarzewski J. (2011b), Finite zeros of positive continuous-time systems, Bull. Pol. Acad. Sci. Tech., 59(3), 293-298.

27. Valcher M.E. (1977), On the initial stability and asymptotic behaviour of 2D positive systems, IEEE Trans. on Circuits and Systems - I, 44(7), 602-613.

28. Wolovich W.A. (1974), Linear Multivariable Systems, SpringerVerlag, New York.

Acknowledgment: The studies have been carried out in the framework of work No. S/WE/1/2016 and financed from the funds for science by the Polish Ministry of Science and Higher Education. 